\title{
Habilidades motoras fundamentais, estado nutricional e fatores ambientais associados em crianças indígenas de aldeias do Amazonas e Minas Gerais
}

\author{
Fundamental motor skills, nutritional status, and environmental associated factors in indigenous \\ children of the villages from Amazonas and Minas Gerais \\ Habilidades motoras fundamentales, estado nutricional y factores ambientales asociados en niños \\ indígenas de los pueblos de Amazonas y Minas Gerais
}

Recebido: 09/12/2021 | Revisado: 15/12/2021 | Aceito: 05/01/2022 | Publicado: 08/01/2022

\author{
Marcelo Gonçalves Duarte \\ ORCID: https://orcid.org/0000-0002-7655-8583 \\ Universidade Federal de Mato Grosso do Sul, Brasil \\ E-mail: duarte.marcelo@ufms.br \\ Rodolfo Novellino Benda \\ ORCID: https://orcid.org/0000-0002-9785-8323 \\ Universidade Federal de Pelotas, Brasil \\ E-mail: rodolfobenda@yahoo.com.br
}

\begin{abstract}
Resumo
$\mathrm{O}$ objetivo foi investigar o desempenho em habilidades motoras fundamentais, o estado nutricional e os fatores ambientais associados em crianças indígenas. Participaram 66 crianças indígenas da etnia Sateré-Mawé e Pataxós residentes em aldeias do estado do Amazonas (AM) e Minas Gerais (MG). Os resultados mostraram que crianças indígenas de MG apresentaram maior peso, estatura, IMC e maior desempenho em habilidades de controle de objetos que crianças indígenas do AM. Nas crianças indígenas de MG, os fatores ambientais oferecem mais oportunidades de práticas fisicamente ativas. Fatores ambientais associaram-se de maneira distinta ao desempenho das habilidades motoras de indígenas do AM e MG. Conclui-se que alguns fatores direcionaram para possíveis trajetórias de maior desempenho em habilidades motoras fundamentais, dependendo da inter-relação organismo e ambiente vivenciado.

Palavras-chave: Indígenas; Habilidades motoras fundamentais; Ambiente.
\end{abstract}

\begin{abstract}
The objective was to investigate the performance in fundamental motor skills, nutritional status, and the environmental associated factors of indigenous children. Sixty indigenous children of the Sateré-Mawé and Pataxós ethnic groups residing in the villages of the state of Amazonas (AM) and Minas Gerais (MG) participated of the study. The results showed greater weight, height, BMI and greater performance in object control skills of indigenous children of MG compared to AM. In MG indigenous, environmental factors afforded more opportunities for physically active practices. Environmental factors associated in a different way with the performance of motor skills of indigenous from AM and MG. We conclude that some factors directed to possible trajectories of greater performance in fundamental motor skills, depend on the interaction between the organism and the experienced environment.
\end{abstract}

Keywords: Indigenous; Fundamental motor skills; Environment.

\section{Resumen}

El objetivo fue investigar el desempeño en habilidades motoras fundamentales, estado nutricional y factores ambientales asociados en niños indígenas. Participaron 66 niños indígenas de la etnia Sateré-Mawé y Pataxós, residentes en aldeas del estado de Amazonas (AM) y Minas Gerais (MG). Los resultados mostraron que los niños indígenas de MG tenían mayor peso, talla, IMC y mayor desempeño en las habilidades de control de objetos que los niños indígenas de BF. En los niños indígenas de MG, los factores ambientales ofrecen más oportunidades para prácticas físicamente activas. Los factores ambientales se asociaron claramente con el desempeño de las habilidades motoras de los pueblos indígenas de AM y MG. Se concluye que algunos factores conducen a posibles trayectorias de mayor desempeño en habilidades motoras fundamentales, dependiendo de la interrelación entre organismo y ambiente experimentado.

Palabras clave: Indígena; Habilidades motoras fundamentales; Ambiente. 


\section{Introdução}

O desenvolvimento motor é um processo contínuo de sequência, durante o qual o ser humano evolui de movimentos simples para movimentos regulares, habilidades motoras mais complexas e, finalmente, a adaptação das habilidades devido ao envelhecimento (Barnett et al., 2020; Gallahue et al., 2013). Na investigação do desempenho motor em habilidades motoras, as influências dos ambientes e diferentes culturas são fundamentais, pois sempre estão se relacionando e afetando um ao outro, podendo o indivíduo mudar conforme o ambiente que está inserido e indicando o nível de desenvolvimento de crianças e jovens durante a infância e a adolescência (Connolly, 2000; Dania; Kaioglou, \& Venetsanou, 2020; Valentini et al., 2020).

Crianças utilizam as habilidades motoras fundamentais para explorar o ambiente nos quais estão inseridos, de modo que, a proficiência no desempenho dessas habilidades está relacionada com as diferentes experiências que a crianças vivencia no seu cotidiano (Duarte et al., 2021; Valentini et al., 2020). Assim, ambientes que oferecem condições para atividades menos sedentárias podem realmente favorecer o desenvolvimento motor (Carlos et al., 2021; Valentini et al., 2020). Por isso, não apenas o comportamento da criança, mas também os fatores que afetam o desempenho motor devem ser investigados, pois se a interação organismo e ambiente delineiam possibilidades para continuidades ou mudanças nas trajetórias do desenvolvimento, a experiência de organismos em distintos ambientes pode oportunizar a emergência de diferentes trajetórias no desenvolvimento (Behan et al., 2019; D’Hondt et al., 2019; Elder, 1987; Gottlieb, 1998; Sifuentes \& Dessen, 2007).

Ainda, estudos têm mostrado casos de inadequação do estado nutricional, sobretudo sobrepeso e obesidade, entre crianças indígenas, em especial aquelas que residem próximas a centros urbanos (Capelli \& Koifman, 2001; Orellana, Coimbra, Lourenço, \& Santos, 2006; Ribas, Sganzerla, Zorzatto, \& Philippi, 2001; Santos et al., 2018). Esse cenário pode ser explicado, em parte, pelas mudanças nas práticas alimentares, sobretudo no consumo de alimentos industrializados e na redução da atividade física dessas crianças (Marta et al., 2016; Yamall Orellana, de Souza, \& Ponte de Souza, 2019). Ainda, esse estado nutricional inadequado pode ter implicações negativas não apenas para a saúde, mas também, para o desempenho motor dessas crianças (Behan et al., 2019; Berleze et al., 2007; Borges Neto et al., 2021; D'Hondt et al., 2019; Marramarco et al., 2012; Pazin, Frainer \& Moreira, 2006; Oliveira et al., 2020,).

Portanto, considerando a problemática exposta anteriormente, será que os ambientes das aldeias apresentam as mesmas oportunidades e podem direcionar para desempenhos similares em habilidades motoras fundamentais? Os fatores que direcionam esse desempenho motor são semelhantes entre si? O estado nutricional destas crianças pode afetar o desempenho motor em locomoção e controle de objetos? O presente estudo tem por objetivo investigar o desempenho em habilidades motoras fundamentais e fatores associados em crianças indígenas da etnia Sateré-Mawé e Pataxós residentes em aldeias do estado do Amazonas e Minas Gerais.

\section{Metodologia}

Trata-se de uma pesquisa básica, com abordagem quantitativa e exploratória de campo (Thomas, Nelson \& Silverman, 2015). Participaram do estudo, 66 crianças, 43 crianças indígenas Sateré-Mawé da aldeia no Amazonas (AM) e seus familiares residentes na aldeia Ponta Alegre, pertencente ao município de Barreirinha (AM), e 23 crianças indígenas da aldeia Pataxós em Minas Gerais (MG) entre oito e dez anos e seus respectivos familiares residentes na aldeia fazenda Guarani no município de Carmésia (MG). Independente da região onde residem, as crianças indígenas estavam matriculadas na escola da aldeia de sua respectiva localidade. O projeto foi aprovado pela Comissão Nacional de Ética e Pesquisa (CONEP) sob o processo número CAAE n ${ }^{\circ}$ 62705916.1.0000.5149.

A massa corporal foi determinada em balança digital da marca Plenna®, escalonada em quilos, com precisão de 100 gramas. A estatura foi obtida em estadiômetro de alumínio (Balmak EST - 222) com escala de $1 \mathrm{~mm}$, variando de 0 a $240 \mathrm{~cm}$. A partir destas medidas calculou-se o Índice de Massa Corporal (IMC), e obteve-se o escore Z classificando o estado 
nutricional em: magreza severa (< escore z -3); magreza (> escore z -3 e < escore z -2), eutrófico ( $>$ escore z - 2 e $<$ escore $\mathrm{z}$ +1 ), sobrepeso ( $>$ escore $z+1$ e $<$ escore $z+2$ ), obesidade ( $>$ escore $z+2$ e $<$ escore $z+3$ ) e obesidade grave $(>$ escore $z+3$ ) (World Health Organization, 2007; Sistema de Vigilância Alimentar e Nutricional, 2008).

Foi utilizada uma anamnese para a avaliação das características ambientais, compreendendo questões relacionadas aos espaços vividos da criança bem como seu tempo livre e ações nele realizadas. A anamnese foi respondida pelos pais ou responsáveis das crianças participantes do estudo. A anamnese foi composta por questões (descritivas/múltipla escolha) (Berleze, 2002, Brauner, 2010; Carvalhal, 2007). Para a avaliação do desempenho em habilidades fundamentais foi utilizado o Test of Gross Motor Development - Second Edition, desenvolvido por Ulrich (2000) e validado no Brasil por Valentini (2012). O TGMD-2 é um teste adequado para a faixa etária dos três aos dez anos e 11 meses de idade e consiste numa avaliação normativa das habilidades motoras amplas. O teste encontra-se dividido em duas categorias locomoção e controle de objetos. Para cada habilidade são observados de três a cinco critérios específicos (Ulrich, 2000). Se o desempenho da criança contemplar o critério determinado no item da habilidade, é atribuído um ponto. Caso o critério avaliativo não seja contemplado, é atribuída a pontuação zero. Posteriormente as pontuações dos itens de cada habilidade são somadas, obtendo-se um escore bruto que variam de zero a 48 pontos tanto para a dimensão locomoção quanto para a dimensão controle de objetos (Ulrich, 2000; Valentini, 2012)

A atividade de pesquisa na aldeia indígena Sateré-Mawé em Ponta Alegre na cidade de Barreirinha no estado do Amazonas ocorreu em duas semanas não consecutivas para o alcance da amostra. Em cada semana, dois dias foram destinados à viagem (um dia de ida e outro de retorno), resultando cinco dias de coleta na aldeia. Autorizados pelo líder da aldeia, foi conduzida uma reunião para esclarecer aos responsáveis pelas famílias as atividades que seriam desenvolvidas. No primeiro dos cinco dias, foram realizadas as coletas dos dados referentes ao estado nutricional (antropometria) e da caracterização do ambiente (anamnese) nas residências dos indígenas no formato de entrevista. Para auxiliar nas coletas, um professor da escola, residente e nativo da aldeia Ponta Alegre, colaborou participando das atividades para facilitar a díade com os familiares. Nos demais dias, foram realizadas as avaliações do desempenho em habilidades fundamentais, que ocorreram na escola da aldeia indígena. Na aldeia indígena Pataxó no município de Carmésia estado de Minas Gerais, as coletas foram realizadas em cinco dias. Nos dois primeiros dias foram realizadas as coletas da caracterização do ambiente nas residências dos indígenas. Para auxiliar nas coletas, um professor da escola, residente e nativo da aldeia Pataxó colaborou para facilitar o contato com os familiares. Nos três dias posteriores, foram realizadas as avaliações antropométricas e de desempenho em habilidades motoras fundamentais, que ocorreram na escola da aldeia indígena.

Foram utilizadas as medidas de tendência central (média: M), dispersão (desvio padrão: DP) para idade, estatura, peso, IMC, desempenho em locomoção e controle de objetos. Frequência absoluta e percentual da frequência foram utilizados para a classificação do estado nutricional e características ambientais. Foi utilizado o teste de associação linear por linear com correção de Bonferroni para verificar possíveis diferenças na proporção da classificação do estado nutricional entre os grupos. Para as características ambientais de meninos e meninas indígenas do Amazonas e Minas Gerais recorreu-se pela avaliação dos dados por meio de frequência absoluta $\left(f_{\mathrm{i}}\right)$ e frequência percentual (\%). Foi utilizada a ANOVA two way (grupo x sexo) para comparar desempenho em locomoção e controle de objetos. O tamanho do efeito foi avaliado pelo eta parcial ao quadrado $\left(\eta^{2} \rho\right)$ (Cohen, 1988; Maroco, 2018). Para verificar a influência dos fatores ambientais e do IMC no desempenho em habilidades de controle de objetos de crianças indígenas da aldeia no Amazonas e Minas Gerais utilizou-se da análise de regressão linear múltipla univariada pelo método stepwise, adotando o último modelo para o presente estudo. As análises foram realizadas no programa estatístico Statistical Package for Social Science (SPSS ${ }^{\circledR}$ ), versão 21. A significância adotada foi de $\alpha \leq 0,05$. 


\section{Resultados}

Na Tabela 1, a análise de variância para a idade não indicou efeito principal estatisticamente significante do grupo $\left(F(1,62)=5,479 ; p=0,135 ; \eta^{2} \rho=0,081\right)$, sexo $\left(F(1,62)=0,508 ; p=0,479 ; \eta^{2} \rho=0,008\right)$ ou efeito de interação grupo e sexo $\left(\mathrm{F}(1,62)=0,191 ; \mathrm{p}=0,664 ; \eta_{\rho}^{2}=0,003\right)$. A análise de variância revelou efeito principal significativo do grupo sobre a estatura $\left(\mathrm{F}(1,62)=10,576 ; \mathrm{p}=0,002 ; \eta^{2}{ }_{\rho}=0,146\right)$. Crianças MG apresentaram maior estatura que crianças AM. A análise não indicou efeito principal significante do sexo $\left(F(1,62)=0,364 ; p=0,549 ; \eta^{2}{ }_{\rho}=0,006\right)$ bem como efeito de interação $(F(1,62)=0,324 ; p$ $\left.=0,571 ; \eta^{2} \rho=0,005\right)$. A análise mostrou efeito principal significativo do grupo para o massa corporal $(\mathrm{F}(1,62)=32,427 ; \mathrm{p}<$ 0,001; $\left.\eta_{\rho}^{2}=0,343\right)$, em que indígenas MG apresentaram maior massa corporal que crianças indígenas AM. A análise não indicou efeito principal do sexo $\left(F(1,62)=0,791 ; p=0,377 ; \eta_{\rho}^{2}=0,013\right)$, assim como efeito de interação $(F(1,62)=0,003 ; p=$ 0,995; $\left.\eta_{\rho}^{2}<0,001\right)$. Para o IMC, há efeito principal estatisticamente significativo para o grupo $(\mathrm{F}(1,62)=13,430 ; \mathrm{p}=0,001$; $\left.\eta_{\rho}^{2}=0,178\right)$. Crianças indígenas MG apresentaram maior IMC que crianças indígenas AM. A análise de variância não mostrou efeito principal do sexo $\left(F(1,62)=0,700 ; p=0,406 ; \eta^{2} \rho=0,011\right)$, bem como efeito de interação $(F(1,62)=2,089 ; p=0,153$; $\left.\eta^{2} \rho=0,033\right)$ para o IMC.

Tabela 1. Estatura, peso, IMC e classificação do estado nutricional crianças indígenas AM e MG.

\begin{tabular}{|c|c|c|c|c|c|c|}
\hline & \multicolumn{3}{|c|}{$\mathbf{A M}$} & \multicolumn{3}{|c|}{ MG } \\
\hline & $\begin{array}{l}\text { Meninos } \\
\text { M (DP) }\end{array}$ & $\begin{array}{l}\text { Meninas } \\
\text { M (DP) }\end{array}$ & $\begin{array}{c}\text { Total } \\
\text { M (DP) }\end{array}$ & $\begin{array}{l}\text { Meninos } \\
\text { M (DP) }\end{array}$ & $\begin{array}{l}\text { Meninas } \\
\text { M (DP) }\end{array}$ & $\begin{array}{c}\text { Total } \\
\text { M (DP) }\end{array}$ \\
\hline Idade (anos) & $8,52(0,80)$ & $8,30(0,10)$ & $8,41(0,62)$ & $8,88(0,94)$ & $8,83(0,89)$ & $8,86(0,89)$ \\
\hline Peso (kg) & $22,69(2,53)$ & $21,90(2,61)$ & $22,29(2,57)^{\mathrm{a}}$ & $27,77(6,23)$ & $26,97(3,36)$ & $27,28(4,58)^{\mathrm{a}}$ \\
\hline $\operatorname{IMC}\left(\mathrm{kg} / \mathrm{m}^{2}\right)$ & $15,08(2,71)$ & $14,77(1,36)$ & $14,93(1,63)^{\mathrm{a}}$ & $15,81(2,59)$ & $16,96(2,56)$ & $16,51(2,53)^{\mathrm{a}}$ \\
\hline Clas. estado nutricional & $f i(\%)$ & $f i(\%)$ & $f i(\%)$ & $f i(\%)$ & $f i(\%)$ & $f i(\%)$ \\
\hline Magreza & $3(13)$ & $1(5)$ & $4(9,3)$ & - & $4(17,4)$ & $4(17,4)$ \\
\hline Eutrófico & $16(69,6)$ & $19(95)$ & $35(81,4)$ & $8(88,9)$ & $5(21,7)$ & $13(56,5)$ \\
\hline Sobrepeso & $1(4,3)$ & - & $1(2,3)$ & - & $5(21,7)$ & $5(21,7)$ \\
\hline
\end{tabular}

Legenda: AM: indígenas do Amazonas; MG: indígenas de Minas Gerais; M: média; DP: desvio padrão; fi: frequência absoluta; \%: frequência percentual relativa. Diferença significativa ( ${ }^{\mathrm{a}}$ : $\left.\mathrm{AM}<\mathrm{MG}\right)$. Fonte: Autores.

Ainda na Tabela 1, o teste de associação linear por linear grupo e classificação do estado nutricional com a correção de Bonferroni, não indicou diferença significativa nas proporções $\left(\chi^{2}=0,940 \mathrm{p}=0,332\right.$; Cramer's V $\left.=0,358\right)$. Observou-se muitas crianças indígenas MG com magreza acentuada, magreza e sobrepeso. Para o estado nutricional não foi observada diferença significante nas proporções $\left(\chi^{2}=3,674 \mathrm{p}=0,055\right.$; Cramer's $\left.\mathrm{V}=0,328\right)$ do estado nutricional.

Na Tabela 2, em relação as características ambientais das crianças, observou-se que a maioria de crianças indígenas de ambos os grupos residiam com mais de dois adultos em casa e possuíam mais de quatro irmãos. Referente ao tempo livre, a maioria das crianças indígenas permaneciam mais de duas horas com tempo livre, assim como, a maioria brincava mais na rua. Crianças indígenas AM dormiam, em sua maioria, de nove a 12 horas, enquanto em maior número, crianças indígenas MG dormiam menos de nove horas diárias. A maior parte dos indígenas realizava tarefas domésticas, entretanto a maior parte de meninos indígenas AM não realizavam. Em sua totalidade, crianças indígenas AM não possuíam televisão e computador, enquanto a maior parte das crianças indígenas MG permaneciam menos de duas horas na televisão e não possuíam computador. Quanto à realização de atividades finas dentro do lar, a maioria das crianças indígenas AM não realizavam. De modo contrário, em maior número, crianças indígenas MG realizavam essa tarefa. A totalidade dos indígenas permanecia até quatro horas na escola e deslocava de maneira não motorizada para escola. A maioria realizava atividades com ênfase em 
controle de objetos. Na totalidade, crianças indígenas AM não praticavam esporte, enquanto a maioria das crianças indígenas MG praticava algum esporte. Quanto ao sexo que mais brincavam, a maior parte dos indígenas optava por brincar com crianças do mesmo sexo. Ainda na Tabela 2, em maioria, crianças indígenas AM optavam por brincar com crianças da mesma idade, enquanto meninas indígenas de MG brincavam com crianças da mesma idade e meninos brincavam com crianças mais velhas. Pais de indígenas em maior parte realizavam atividades físicas. A maior parte de mães de crianças indígenas AM não realizava atividades físicas, do mesmo modo que mães de meninas indígenas MG. Entretanto, a maioria das mães de meninos indígenas MG realizavam atividades físicas.

Tabela 2. Características ambientais de crianças indígenas AM e MG.

\begin{tabular}{|c|c|c|c|c|c|c|}
\hline & \multicolumn{3}{|c|}{$\mathbf{A M}$} & \multicolumn{3}{|c|}{ MG } \\
\hline & $\begin{array}{c}\text { Meninos } \\
\text { fi }(\%)\end{array}$ & $\begin{array}{c}\text { Meninas } \\
\text { fi }(\%)\end{array}$ & $\begin{array}{r}\text { Total } \\
\text { fi } i(\%)\end{array}$ & $\begin{array}{c}\text { Meninos } \\
\text { fi }(\%)\end{array}$ & $\begin{array}{c}\text { Meninas } \\
\text { fi } i(\%)\end{array}$ & $\begin{array}{l}\text { Total } \\
\text { fi }(\%)\end{array}$ \\
\hline \multicolumn{7}{|l|}{ Adultos em casa } \\
\hline$\leq 2$ adultos & $11(47,8)$ & $5(25)$ & $16(39,5)$ & $2(22,2)$ & $3(21,4)$ & $5(21,7)$ \\
\hline$>2$ adultos & $12(52,2)$ & $15(75)$ & $27(60,5)$ & $7(77,8)$ & $11(78,6)$ & $18(78,3)$ \\
\hline \multicolumn{7}{|l|}{ Irmãos em casa } \\
\hline$\leq 2$ irmãos & $3(13)$ & - & $3(7)$ & $1(11,1)$ & $2(14,2)$ & $3(13)$ \\
\hline $3 \overline{\mathrm{a}} 4$ irmãos & $6(26,1)$ & $6(30)$ & $12(27,9)$ & $2(22,2)$ & $6(42,9)$ & $8(34,8)$ \\
\hline$>4$ irmãos & $14(60,9)$ & $14(70)$ & $28(65,1)$ & $6(66,7)$ & $6(42,9)$ & $12(52,2)$ \\
\hline \multicolumn{7}{|l|}{ Tempo livre } \\
\hline$\leq 1$ hora & $3(13)$ & $3(15)$ & $6(14)$ & $1(11,1)$ & $2(14,3)$ & $3(13)$ \\
\hline 1 a 2 horas & $5(21,7)$ & $2(10)$ & $7(16,3)$ & $1(11,1)$ & $4(28,3)$ & $5(21,7)$ \\
\hline$>2$ horas & $15(65,3)$ & $15(75)$ & $30(69,8)$ & $7(77,8)$ & $8(57,1)$ & $15(65,2)$ \\
\hline \multicolumn{7}{|l|}{ Onde mais brinca } \\
\hline Casa & $3(13)$ & $3(15)$ & $6(14)$ & $1(11,1)$ & $6(42,9)$ & $7(30,4)$ \\
\hline Rua & $13(56,5)$ & $13(65)$ & $26(60,5)$ & $5(55,6)$ & $7(50)$ & $12(52,2)$ \\
\hline Outros & $7(30,4)$ & $4(20)$ & $11(25,6)$ & $3(33,4)$ & $1(7,1)$ & $4(17,4)$ \\
\hline \multicolumn{7}{|l|}{ Tempo que dorme } \\
\hline$\leq 9$ horas & $2(8,7)$ & $12(60)$ & $14(32,6)$ & $6(66,7)$ & $8(57,1)$ & $14(60,9)$ \\
\hline 9 a 12 horas & $10(43,5)$ & $7(35)$ & $17(39,5)$ & $1(11,1)$ & $3(21,4)$ & $4(17,4)$ \\
\hline$>12$ horas & $11(47,8)$ & $1(5)$ & $12(27,9)$ & $2(22,2)$ & $3(21,4)$ & $5(21,7)$ \\
\hline \multicolumn{7}{|l|}{ Tarefas domésticas } \\
\hline 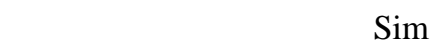 & $6(26,1)$ & $16(80)$ & $22(51,2)$ & $7(77,8)$ & $11(78,6)$ & $18(78,3)$ \\
\hline Não & $17(73,9)$ & $4(20)$ & $21(48,8)$ & $2(22,2)$ & $3(21,4)$ & $5(21,7)$ \\
\hline \multicolumn{7}{|l|}{ Tempo de televisão } \\
\hline Não possui & $23(100)$ & $20(100)$ & $43(100)$ & $3(33,3)$ & $3(21,4)$ & $6(26,1)$ \\
\hline$\leq 2$ horas & - & - & - & $5(55,6)$ & $10(71,4)$ & $15(65,2)$ \\
\hline$>2$ horas & - & - & - & $1(11,1)$ & $1(4,3)$ & $2(8,7)$ \\
\hline \multicolumn{7}{|l|}{ Tempo no computador } \\
\hline Não possui & $23(100)$ & $20(100)$ & $43(100)$ & $8(88,9)$ & $12(85,7)$ & $20(87)$ \\
\hline$\leq 2$ horas & - & - & - & $1(11,1)$ & $2(14,3)$ & $3(13)$ \\
\hline$>2$ horas & - & - & - & - & - & - \\
\hline \multicolumn{7}{|l|}{ At. Motoras finas } \\
\hline Sim & $6(26,1)$ & $12(60)$ & $18(41,9)$ & $7(77,8)$ & $12(85,7)$ & $19(82,6)$ \\
\hline Não & $17(73,9)$ & $8(40)$ & $25(58,1)$ & $2(22,2)$ & $2(14,3)$ & $4(17,4)$ \\
\hline \multicolumn{7}{|l|}{ Tempo na escola } \\
\hline$\leq 4$ horas & $23(100)$ & $20(100)$ & $43(100)$ & $9(100)$ & $14(100)$ & $23(100)$ \\
\hline$>4$ horas & - & - & - & - & - & - \\
\hline \multicolumn{7}{|l|}{ Atividades fora do lar } \\
\hline Ativ. locomotoras & $9(39,1)$ & $10(50)$ & $19(44,2)$ & $1(11,1)$ & $6(42,9)$ & $7(30,4)$ \\
\hline Ativ.manipulativas & $14(60,9)$ & $10(50)$ & $29(55,8)$ & $8(88,9)$ & $8(57,1)$ & $16(69,6)$ \\
\hline \multicolumn{7}{|l|}{ Deslocamento escola } \\
\hline Não motorizado & $23(100)$ & $20(100)$ & $43(100)$ & $9(100)$ & $14(100)$ & $23(100)$ \\
\hline Motorizado & - & - & - & - & - & - \\
\hline
\end{tabular}




\begin{tabular}{|c|c|c|c|c|c|c|}
\hline \multicolumn{7}{|l|}{ Pratica esporte } \\
\hline Sim & - & - & - & $7(77,8)$ & $9(64,3)$ & $16(69,6)$ \\
\hline Não & $23(100)$ & $20(100)$ & $43(100)$ & $2(22,2)$ & $5(35,7)$ & $7(30,4)$ \\
\hline \multicolumn{7}{|l|}{ Sexo que brinca } \\
\hline Mesmo sexo & $20(87)$ & $18(90)$ & $38(88,4)$ & $7(77,8)$ & $10(71,4)$ & $17(73,9)$ \\
\hline Sexo oposto & $3(13)$ & $2(10)$ & $5(11,6)$ & $2(22,2)$ & $4(28,6)$ & $6(26,1)$ \\
\hline \multicolumn{7}{|l|}{ Idade que brinca } \\
\hline Mais novos & $9(39,1)$ & $1(5)$ & $10(23,3)$ & $1(11,1)$ & $2(14,3)$ & $3(13)$ \\
\hline Mesma idade & $14(60,9)$ & $18(90)$ & $32(74,4)$ & $3(33,3)$ & $7(50)$ & $10(43,5)$ \\
\hline Mais velhos & - & $1(5)$ & $1(2,3)$ & $5(55,6)$ & $5(35,7)$ & $10(43,5)$ \\
\hline \multicolumn{7}{|l|}{ Pai realiza atividade física } \\
\hline Sim & $15(65,2)$ & $11(55)$ & $26(60,5)$ & $7(77,8)$ & $9(64,3)$ & $16(69,6)$ \\
\hline Não & $8(34,8)$ & $9(45)$ & $17(39,5)$ & $2(22,2)$ & $5(35,7)$ & $7(30,4)$ \\
\hline \multicolumn{7}{|l|}{ Mãe realiza atividade física } \\
\hline Sim & $8(34,8)$ & $5(25)$ & $13(30,2)$ & $6(66,7)$ & $5(35,7)$ & $11(47,8)$ \\
\hline Não & $15(65,2)$ & $15(75)$ & $30(69,8)$ & $3(33,3)$ & $9(64,3)$ & $12(52,2)$ \\
\hline
\end{tabular}

Legenda: AM: indígenas do Amazonas; MG: indígenas de Minas Gerais; fi: frequência absoluta; \%: percentual da frequência; $\leq$ : menor igual; >: maior. Fonte: Autores.

Para o desempenho em habilidades motoras fundamentais, a análise de variância two way, não revelou efeito principal significante do grupo $\left(F(1,62)=0,898 ; p=0,347 ; \eta^{2} \rho=0,014\right)$, sexo $\left(F(1,62)=1,364 ; p=0,247 ; \eta^{2} \rho=0,022\right)$ bem como efeito de interação $\left(F(1,62)=0,366 ; p=0,547 ; \eta^{2}{ }_{\rho}=0,006\right)$ no desempenho em locomoção (Tabela 3).

Tabela 31. Desempenho em locomoção e controle de objetos crianças indígenas AM e MG.

\begin{tabular}{lcccccc}
\hline & \multicolumn{3}{c}{ AM } & & \multicolumn{2}{c}{ MG } \\
\cline { 2 - 7 } Desempenho Motor & Meninos & Meninas & Total & Meninos & Meninas & Total \\
M (DP) & M (DP) & M (DP) & M (DP) & M (DP) & M (DP) \\
\hline Locomoção & $40,17(4,09)$ & $38,20(5,09)$ & $39,25(4,63)$ & $40,47(3,53)$ & $39,89(3,40)$ & $40,18(3,39)$ \\
Controle de objetos & $36,18(6,11)$ & $33,49(4,26)$ & $34,83(5,45)^{\mathrm{a}}$ & $42,55(2,29)$ & $42,64(3,05)$ & $42,60(2,72)^{\mathrm{a}}$ \\
\hline
\end{tabular}

Legenda: AM: indígenas do Amazonas; MG: indígenas de Minas Gerais; M: média; DP: Desvio padrão; diferença significativa (a: AM < MG). Fonte: Autores.

A análise de variância indicou efeito principal significativo do grupo $\left(F(1,62)=40,405 ; \mathrm{p}<0,001 ; \eta^{2} \rho=0,395\right)$ no desempenho em habilidades de controle de objetos. Crianças indígenas MG apresentaram maior desempenho em controle de objetos do que crianças indígenas AM. A análise não indicou efeito principal significante do sexo $(F(1,62)=1,166 ; p=0,284$; $\left.\eta_{\rho}^{2}=0,018\right)$, assim como efeito de interação $\left(F(1,62)=1,326 ; p=0,254 ; \eta^{2}{ }_{\rho}=0,021\right)$ para o desempenho em controle de objetos (Tabela 3).

Na Tabela 4, são apresentadas somente as variáveis que mostraram associação significativa com o desempenho das crianças na locomoção. Nas crianças indígenas AM, a análise de regressão linear mostrou um modelo significativo $(\mathrm{F}(3,38)=$ 18,057; $\left.\mathrm{p}<0,001 ; \mathrm{r}_{\mathrm{a}}^{2}=0,619\right)$ e explicou uma proporção de $62 \%$ da variabilidade do desempenho em locomoção destas crianças. Para as crianças indígenas MG, o modelo de regressão mostrou-se significativo $\left(\mathrm{F}(1,20)=12,837 ; \mathrm{p}=0,002 ; \mathrm{r}^{2}{ }_{\mathrm{a}}=\right.$ 0,350) e explicou uma proporção de $35 \%$ da variabilidade do desempenho em locomoção deste grupo de crianças. 
Tabela 42. Fatores associados ao desempenho motor em locomoção de crianças indígenas AM e MG.

\begin{tabular}{|c|c|c|c|c|c|}
\hline Grupo & Fatores & $\boldsymbol{B}$ & $\boldsymbol{\beta}$ & $\mathbf{t}$ & p \\
\hline \multirow{3}{*}{$\mathbf{A M}$} & Tempo livre $>2$ horas $^{\mathrm{a}}$ & 5,968 & 0,598 & 5,326 & $<0,001$ \\
\hline & Brinca mais na rua (fora de casa) ${ }^{\mathrm{b}}$ & 3,071 & 0,292 & 2,950 & 0,005 \\
\hline & Atividades manipulativas $^{\mathrm{c}}$ & $-2,437$ & $-0,264$ & $-2,377$ & 0,023 \\
\hline MG & Tempo livre $>2$ horas $^{\mathrm{a}}$ & 4,292 & 0,616 & 3,583 & 0,002 \\
\hline
\end{tabular}

Legenda: AM: indígenas do Amazonas ( ${ }^{\mathrm{a}}$ categoria de referência: tempo livre $\leq 1$ hora; ${ }^{\mathrm{b}}$ categoria de referência: brinca mais em casa; ${ }^{c}$ categoria de referência: locomotoras); MG: indígenas de Minas Gerais ( ${ }^{\mathrm{a}}$ categoria de referência: tempo livre $\leq 1$ hora). Fonte: Autores.

$\mathrm{Na}$ Tabela 5, quanto às crianças indígenas $\mathrm{AM}$, o modelo mostrou-se significativo $\left(\mathrm{F}(1,40)=9,265 ; \mathrm{p}<0,001 ; \mathrm{r}^{2}{ }_{\mathrm{a}}=\right.$ 0,288) e explicou uma proporção de $29 \%$ da variabilidade do controle de objetos. Para crianças indígenas MG, o modelo mostrou-se significativo $\left(\mathrm{F}(2,19)=8,972 ; \mathrm{p}=0,002 ; \mathrm{r}_{\mathrm{a}}{ }_{\mathrm{a}}=0,420\right)$ e explicou $42 \%$ da variabilidade do desempenho em controle de objetos de crianças indígenas MG.

Tabela 5. Fatores associados ao desempenho em controle de objetos de crianças indígenas AM e MG.

\begin{tabular}{clcccc}
\hline Grupo & \multicolumn{1}{c}{ Fatores } & $\mathbf{B}$ & $\boldsymbol{\beta}$ & $\mathbf{t}$ & $\mathbf{p}$ \\
\hline $\mathbf{A M}$ & Tempo livre $>2$ horas $^{\mathrm{a}}$ & 5,937 & 0,506 & 3,850 & $<0,001$ \\
& & & & & \\
& & & & & \\
\multirow{2}{*}{$\mathbf{M G}$} & Tempo livre $>2$ horas $^{\mathrm{a}}$ & 3,260 & 0,582 & 3,466 & 0,002 \\
& $\leq 2$ horas de computador & $-4,329$ & $-0,547$ & $-3,254$ & 0,004 \\
\hline
\end{tabular}

Legenda: AM: indígenas do Amazonas ( ${ }^{\mathrm{a}}$ categoria de referência: tempo livre $\leq 1$ hora); MG: indígenas de Minas Gerais ( ${ }^{\mathrm{a}}$ categoria de referência: tempo livre $\leq 1$ hora; ${ }^{b}$ categoria de referência: não possui computador). Fonte: Autores.

\section{Discussão}

Embora estudos apresentem altas prevalências de déficit estatural em indígenas (Menegolla et al., 2006; Orellana et al., 2006), crianças indígenas MG apresentaram maior estatura, peso e IMC que crianças indígenas AM. O menor peso, estatura e IMC de crianças indígenas do Norte são registrados por dados do Sistema de Informação da Atenção à Saúde Indígena (SIASI, 2019), em que reportaram que crianças indígenas residentes no norte do Brasil são as crianças com menor peso, estatura e IMC.

No presente estudo, observou-se muitas crianças indígenas MG nos dois extremos da curva de estado nutricional, o que demonstrou a inadequação alimentar que vem se tornando hábito nas aldeias indígenas da região. Devido à proximidade da aldeia a centros urbanos, a população indígena possui cada vez mais acesso à alimentação inadequada, resultando no aumento de consumo de alimentos industrializados. Estudos relatam casos de sobrepeso e obesidade entre crianças indígenas, em especial em populações indígenas que residem próximas a centros urbanos (Capelli \& Koifman, 2001; Orellana et al., 2006; Ribas et al., 2001; Santos et al., 2018). Santos et al. (2018) revelaram que foram identificadas 19,4\% crianças indígenas com sobrepeso, resultado que pode estar relacionado às mudanças nas práticas alimentares, pois o contato com a civilização urbana levou essa população a consumir alimentos industrializados, assim como a uma redução da atividade física, pois o que antes era obtido pela caça, pesca e agricultura de subsistência, passou a ser adquirido pelo trabalho remunerado nos centros urbanos ou na produção e venda de artesanatos (de Moura, Batista, \& Moreira, 2010; Yamall Orellana et al., 2019).

Os resultados demonstraram semelhanças entre as características ambientais onde residem indígenas AM e MG, entretanto, algumas das características ambientais distintas chamaram a atenção. Crianças indígenas MG dormiam menos tempo, por volta de nove horas, o que pode estar relacionado ao fato de que, nesta aldeia indígena, todas as casas possuíam energia elétrica, possibilitando que as crianças permanecerem mais tempo acordadas dentro de suas residências. Essa relação 
fica mais evidente quando se observa que a maioria das crianças indígenas MG permanecia, embora por pouco tempo (menos de duas horas), em frente à televisão e realizavam atividades de motricidade fina dentro da residência. A realização de atividades de motricidade fina, como a produção de artesanato, é bastante incentivada por pais e mães dessas crianças, pois é um recurso para o sustento familiar, principalmente em festejos que ocorrem na aldeia. Coelho (2011), ao investigar indígenas Pataxós da aldeia residentes próximos à cidade de Carmésia/MG, revelou que, embora a maior fonte de renda das famílias devia-se à produção, venda e revenda de artesanato, algumas famílias indígenas apresentavam maior fonte de renda, devido ao trabalho realizado em comércios na cidade, possibilitando-os a disporem dentro de suas residências produtos industrializados como televisores, geladeiras, micro-ondas, máquinas de lavar roupa, aparelhos de som, dvd's, bicicletas e computadores, assim como alguns veículos automotores. Característica ambiental totalmente distinta verificou-se na aldeia do Amazonas, pois a totalidade das residências não possuía energia elétrica, do mesmo modo que não possuía televisão ou outros eletrodomésticos. Distinto dos achados do presente estudo quanto às crianças indígenas MG, a maioria dos indígenas no Brasil, principalmente aqueles residentes em aldeias, somente 10\% dispõem de televisão em casa (Instituto Brasileiro de Geografia e Estatística, 2005). Ainda assim, novos levantamentos são necessários, de forma a ter um panorama mais atualizado sobre esta questão. Outra característica distinta entre as aldeias é o fato de que a maioria das crianças indígenas MG praticam alguma modalidade esportiva. A proximidade do ambiente urbano possibilitava que a maioria das crianças indígenas MG participem de projetos esportivos ofertados no ambiente urbano. A maioria deslocava-se de bicicleta até esses projetos.

Os resultados do desempenho em habilidades motoras fundamentais demostram que, crianças indígenas AM e MG apresentaram desempenho em locomoção semelhante, assim como, meninos e meninas apresentaram desempenho similar. Esse achado, pode ser reflexo das atividades que requeiram locomoção entre indígenas de aldeias, seja no estado do Amazonas ou em Minas Gerais, independente do sexo. Embora as aldeias sejam distintas, algumas das práticas vivenciadas pelas crianças se assemelham, como por exemplo, brincar por toda aldeia, com atividades de pega-pega, corrida etc., e até mesmo deslocarem-se de uma "casa" para outra, a fim de convidar seus amigos para brincarem no campo de futebol.

Para o desempenho em controle de objetos, mostram que, crianças indígenas AM apresentaram desempenho inferior as crianças indígenas MG, e meninos apresentaram desempenho em controle de objetos semelhante as meninas. A similaridade do desempenho de meninos e meninas, embora em aldeias as distinções entre comportamentos dos sexos sejam evidentes, parece que nessa faixa etária (oito a dez anos) as atividades que meninos e meninas indígenas realizam não influenciaram na diferença do desempenho em controle de objetos entre sexos.

$\mathrm{O}$ maior desempenho em controle de objetos de crianças indígenas MG em relação às crianças indígenas $\mathrm{AM}$ pode estar vinculado a alguns comportamentos vivenciados no ambiente em que residem. Embora a maioria das crianças indígenas MG participem de atividades esportivas, e estudos têm indicado que práticas esportivas orientadas contribuem decisivamente para o melhor desempenho em habilidades motoras fundamentais (Brauner \& Valentini, 2009; Cotrim, Lemos, Néri Júnior, \& Barela, 2011; D'Hondt et al., 2014; Foweather et al., 2008; Marramarco et al., 2012; Vandorpe et al., 2012), é possível que a participação em esportes seja apenas um dos aspectos que expressem o maior desempenho em controle de objetos de crianças indígenas MG em relação às crianças indígenas AM. Parece que a inter-relação estabelecida entre organismo e ambiente físico e sociocultural vivenciado contribua para um maior desempenho em controle de objetos de crianças indígenas MG.

Permanecer mais tempo livre direcionou para um melhor desempenho em locomoção e controle de objetos em crianças indígenas AM e MG, e brincar mais na rua (fora de casa) direcionou para o desempenho em locomoção de crianças indígenas AM. Uma das características presentes nas aldeias indígenas é o tempo livre disponibilizado para as crianças. Brincadeiras observadas junto às crianças indígenas Xikrin revelam que as crianças não têm hora para brincar, elas brincam o tempo todo, pois permanecem com mais tempo livre para brincar por toda aldeia (Cohn, 2002). As crianças brincam com companheiros do mesmo sexo, mesma faixa etária, passam maior tempo brincando (Grosso, 2009). A criança indígena aprende 
experimentando, vivendo o dia da aldeia e, acima de tudo, acompanhando a vida dos mais velhos, imitando, criando, inventando, sendo que o ambiente familiar, composto pelo grupo de parentesco, oferece a liberdade e a autonomia necessária para esse experimentar e criar infantil (Nascimento \& Vieira 2015). De fato, crianças que possuem mais oportunidades de vivenciar práticas motoras possivelmente apresentarão maior desempenho em habilidades motoras fundamentais, pois dedicarão maior tempo em brincadeiras, atividades mais ativas que aquelas crianças que dedicam mais tempo brincando dentro de casa.

Permanecer menos de duas horas no computador associa-se negativamente com o desempenho em controle de objetos de crianças indígenas MG. Embora por pouco tempo, crianças permaneciam no computador, o que de certa maneira influenciou negativamente no seu desempenho. Comportamentos sedentários (ver televisão ou outro meio audiovisual) vêm sendo reportados como um dos responsáveis para o baixo desempenho em habilidades motoras fundamentais (Cadoret, Bigras, Lemay, Lehrer, \& Lemire, 2018; Stabelini Neto, Mascarenhas, Nunes, Lepre, \& Campos, 2004; Stodden et al., 2008; Webster, Martin, \& Staiano, 2019), uma vez que a falta de exploração motora tende a reduzir o repertório de movimentos, sendo prejudicial ao desenvolvimento e à saúde.

\section{Considerações Finais}

O estado nutricional da maioria das crianças indígenas das aldeias do Amazonas e Minas Gerais apresentou-se dentro da normalidade. Crianças indígenas das aldeias mostraram desempenho em habilidades de locomoção similares, e crianças indígenas de Minas Gerais apresentaram maior desempenho em habilidades de controle de objetos que as crianças indígenas do Amazonas. Características ambientais vivenciadas por crianças indígenas das aldeias do Amazonas e Minas Gerais se assemelham e direcionam para o envolvimento com ações menos sedentárias. Este achado pode estar vinculado com as ações realizadas pelas crianças, é possível que a prática de esporte realizada pelas crianças indígenas de Minas Gerais possa estar contribuindo para um maior desempenho em habilidades de controle de objetos. A inter-relação estabelecida entre crianças indígenas de Minas Gerais e ambiente físico e sociocultural contribui para um maior desempenho em controle de objetos em relação as crianças indígenas do Amazonas. Fatores associados seja em crianças indígenas de Minas Gerais e do Amazonas foram distintos, mostrando que, embora sejam aldeias, as trajetórias para um maior desempenho em habilidades fundamentais são particulares e resultado da inter-relação entre organismo e ambiente.

Os achados do presente estudo podem direcionar o encaminhamento de intervenções a serem realizadas a fim de otimizar o desempenho em habilidades motoras fundamentais, bem como otimizar o contexto ambiental vivenciado por crianças indígenas. Um maior conhecimento sobre as atividades e o tempo de permanência nessas atividades que as crianças realizam ao longo do tempo seria necessário para uma conclusão mais robusta, caracterizando como limitações do presente estudo. O presente estudo avança ao propiciar um maior suporte no entendimento de uma população pouco investigada e com acesso extremamente particular, assim como, para uma melhor compreensão de quais os fatores podem estar em prioridade para um maior desempenho em habilidades motoras fundamentais. Ainda são necessários estudos que investiguem de maneira mais minuciosa quais são as atividades e a temporalidade das atividades que essas crianças realizam ao longo do tempo.

\section{Referências}

Barnett, L. M., Hnatiuk, J. A., Salmon, J., \& Hesketh, K. D. (2019). Modifiable factors which predict children's gross motor competence: a prospective cohort study. International Journal of Behavioral Nutrition and Physical Activity, 16, 129. https://doi.org/10.1186/s12966-019-0888-0

Behan, S., Belton, S., Peers, C., O’Connor. N. E., \& Issartel, J. (2019). Moving Well-Being Well: Investigating the maturation of fundamental movement skill proficiency across sex in Irish children aged five to twelve. Journal of Sports Sciences, 37(22), 2604-2612. https://doi.org/10.1080/02640414.2019.1651144

Brauner, L. M., \& Valentini, N. C. (2009). Análise do desempenho motor de crianças participantes de um programa de atividades físicas. Revista da Educação Física/UEM, 20(2), 205-216. https://doi.org/10.4025/reveducfis.v20i2.6070 
Borges Neto, J. de S., Benda, R. N., Novais, R. L. R., Bicalho, J. M. F, Romano, M. C. C, \& Lamonier, J. A. (2021). Desempenho motor de habilidades fundamentais de estudantes de ambos os sexos do Ensino Fundamental. Research, Society and Development, 10(5), e361101523109. https://dx.doi.org/10.33448/rsd-v10i15.23109

Cadoret, G., Bigras, N., Lemay, L., Lehrer, J., \& Lemire, J. (2018). Relationship between screen-time and motor proficiency in children: a longitudinal study. Early Child Development and Care, 188(2), 231-239. https://doi.org/10.1080/03004430.2016.1211123

Capelli, J. de C. S., \& Koifman, S. (2001). Avaliação do estado nutricional da comunidade indígena Parkatêjê, Bom Jesus do Tocantins, Pará, Brasil. Cadernos de Saúde Pública, 17(2), 433-437. https://doi.org/10.1590/S0102-311X2001000200018

Carlos, F. de M., Borges Neto, J. de S., Bicalho, J. M. F., Oliveira, G. H., Campos, C. G., Bila, W. C., Lamounier, J. A., \& Romano, M. C. C. (2021). Adolescentes socialmente menos favorecidos estão mais sujeitos ao sedentarismo? uma revisão sistemática.

Research, Society and Development, 10(14), e305101422048. https://doi.org/10.33448/rsd-v10i14.22048

Coelho, L. S. Infância, aprendizagem e cultura: as crianças pataxó e as práticas sociais do guarani. (2011). Dissertação (Mestrado em Lazer) - Escola de Educação Física, Fisioterapia e Terapia Ocupacional, Universidade Federal de Minas Gerais, Belo Horizonte.

Cohn, C. (2002). A experiência da infância e o aprendizado entre os xikrin. In: lopes da silva, a.; macedo, a. V. L. S.; nunes, a. (orgs.). Crianças indígenas: ensaios antropológicos. São Paulo: global.

Connolly, K. (2000). Desenvolvimento motor: Passado, preente e futuro. Rev. Paul. Educ. Fis., São Paulo, Supl.3, 6-15.

Cohen, J. (1988). Statistical power analysis for the behavioral sciences (2nd ed., Vol. 2). London: Lawrence Erlbaum Associates.

Cotrim, J. R., Lemos, A. G., Néri Júnior, J. E., \& Barela, J. A. (2011). Desenvolvimento de habilidades motoras fundamentais em Crianças com diferentes contextos escolares. Revista Da Educacao Fisica, 22(4), 523-533. https://doi.org/10.4025/reveducfis.v22i4.12575

D’Hondt, E., Deforche, B., Gentier, I., Verstuyf, J., Vaeyens, R., De Bourdeaudhuij, I., \& Lenoir, M. (2014). A longitudinal study of gross motor coordination and weight status in children. Obesity, 22(6), 1505-1511. https://doi.org/10.1002/oby.20723

D’Hondt E, Venetsanou F, Kambas A, Lenoir M. (2019). Motor Competence Levels in Young Children: A Cross-Cultural Comparison Between Belgium and Greece. Journal of Motor Learning and Development, 7(3), 289-306. https://doi.org/10.1123/jmld.2018-0044

de Moura, P. G., Batista, L. R. V., \& Moreira, E. A. M. (2010). População indígena: Uma reflexão sobre a influência da civilização urbana no estado nutricional e na saúde bucal. Revista de Nutricao, 23(3), 459-465. https://doi.org/10.1590/S1415-52732010000300013

Duarte, G. D., Nobre, G. C., Gomes, T. V. B., \& Benda, R. N. (2021). Fundamental Motor Skill Performance of Indigenous and Nonindigenous Children. Journal of Motor Learning and Development, 9, 14 - 27 https://doi.org/10.1123/jmld.2019-0050

Elder Jr., G. H. (1996). Human lives in changing societies: Life course and developmental insights. In R. B. Cairns, g. H. Elder jr., \& e. J. Costello (Eds.), Developmental science (pp. 31-62). New York: Cambridge University Press.

Foweather, L., McWhannell, N., Henaghan, J., Lees, A., Stratton, G., \& Batterham, A. M. (2008). Effect of a 9-wk. After-school multiskills club on fundamental movement skill proficiency in 8- to 9-yr.-old children: An exploratory trial. Perceptual and Motor Skills, 106(3), 745-754. https://doi.org/10.2466/PMS.106.3.745-754

Gallahue, D. L.; Ozmun, J. C.; Goodway, J. D. (2013). Compreendendo o desenvolvimento motor: bebês, crianças, adolescentes e adultos. Porto Alegre: Artmed.

Gottlieb, G. (1998). Normally occurring environmental and behavioral influences on gene activity: From central dogma to probabilistic epigenesis. Psychological Review, 105(4), 792-802. https://doi.org/Doi 10.1037//0033-295x.105.4.792-802

Grosso, Y. (2005). Pexe oxemoarai: brincadeiras infantis entre os índios Parakanã (Universidade de São Paulo). https://doi.org/10.11606/T.47.2005.tde21032006-105319

Instituto Brasileiro de geografia e estatística (IBGE). Tendências demográficas: Uma análise dos indígenas com base nos resultados da amostra dos Censos demográficos 1991 e 2000. Brasília. 2005.

Marramarco, C. A., Krebs, R. J., Valentini, N. C., Da Silva Ramalho, M. H., Dos Santos, J. O. L., \& Nobre, G. C. (2012). Crianças desnutridas pregressas, com sobrepeso e obesas apresentam desempenho motor pobre. Revista Da Educacao Fisica, 23(2), 175-182. https://doi.org/10.4025/reveducfis.v23i2.13002

Maroco, J. (2014). Análise Estatística com o SPSS Statistics (6th ed.). Pêro Pinheiro: Report Number.

Marta, C., Casanova, N., Fonseca, T., Vila-Chã, C., Esteves, P. T., Carvalhal, M. I. M., ... Marinho, D. A. (2016). Efeito das variáveis biológicas, socioculturais e motoras na prestação do lançamento em crianças pré-pubertárias. Motricidade, 12(1), 83-95. https://doi.org/10.6063/motricidade.6294

Menegolla, I. A., Drachler, M. D. L., Rodrigues, I. H., Schwingel, L. R., Scapinello, E., Pedroso, M. B., \& De Carvalho Leite, J. C. (2006). Nutritional status and social determinants of child height in the Guarita Indigenous Territory, Southern Brazil. Cadernos de Saude Publica, $22(2)$, 395-406. https://doi.org/10.1590/S0102-311X2006000200017

Nascimento, A. C.; Vieira, C. M. N. (2015). O índio e o espaço urbano: breves considerações sobre o contexto indígena na cidade. Revista Cordis História: cidade, esporte e lazer, São Paulo, n. 14, p.118-136.

Niemistö, D., Finni, T., Haapala, E. A., Cantell, M., Korhonen, E., \& Sääkslahti A. (2019). Environmental correlates of motor competence in children-The Skilled Kids Study. International Journal of Environmental Research and Public Health, 16(11), 1989. https://doi.org/10.3390/ijerph16111989 
Research, Society and Development, v. 11, n. 1, e35811124549, 2022

(CC BY 4.0) | ISSN 2525-3409 | DOI: http://dx.doi.org/10.33448/rsd-v11i1.24549

Orellana, J. D. Y., Coimbra, C. E. A., Lourenço, A. E. P., \& Santos, R. V. (2006). Estado nutricional e anemia em crianças Suruí, Amazônia, Brasil. Jornal de Pediatria, 82(5), 383-388. https://doi.org/10.2223/JPED.1528

Oliveira, V. P. de, Santos, A. A. dos., Silva, a. G. da., Reis, F. P. G. dos., Batista, M. T. S., Pereira, F. de P., Ferreira, L. F., Souza, C. J. F. de., Bruzi, A. T. (2020). Effects of school environments and the resources made available to physical education classes on the motor proficiency of children in a municipality in the south of Minas Gerais. Research, Society and Development, v. 9, n. 11, p. e2039119697, 2020. https://doi.org/10.33448/rsd-v9i11.9697

Orellana, J. D., de Souza, C. C., \& Ponte de Souza, M. L. (2019). Hidden Suicides of the Indigenous People of the Brazilian Amazon: Gender, Alcohol and Familial Clustering. Revista Colombiana de Psiquiatria, 48(3), 133-139. https://doi.org/10.1016/j.rcp.2017.12.001

Pazin, J., Frainer D. E. S., \& Moreira D. (2006). Crianças obesas têm atraso no desenvolvimento motor. Revista digital efdeportes. http://www.efdeportes.com

Ribas, D. L. B., Sganzerla, A., Zorzatto, J. R., \& Philippi, S. T. (2001). Nutrição e saúde infantil em uma comunidade indígena Teréna, Mato Grosso do Sul, Brasil. Cadernos de Saúde Pública, 17(2), 323-331. https://doi.org/10.1590/s0102-311x2001000200007

Santos, A. P., Mazzeti, C. M. da S., Franco, M. do C. P., Santos, N. L. G. O., Conde, W. L., Leite, M. S., \& Castro, T. G. de. (2018). Nutritional status and environmental and health conditions of Pataxó indigenous children, Minas Gerais State, Brazil. Cadernos de Saude Publica, 34(6). https://doi.org/10.1590/0102-311X00165817

Sifuentes, T. R., \& Dessen, M. A. (2007). Desenvolvimento Humano: Desafios para a Compreensão das Trajetórias Probabilísticas Human Development: Challenges for the Comprehension of the Probabilistic Trajectories, 23, 379-386.

Stabelini Neto, A., Mascarenhas, L. P. G., Nunes, G. F., Lepre, C., \& Campos, W. De. (2004). Relação entre fatores ambientais e habilidades motoras básicas em crianças de 6 e 7 anos. Revista Mackenzie de Educação Física e Esporte, 3(3), 135-140.

Stodden, D. F., Goodway, J. D., Langendorfer, S. J., Roberton, M. A., Rudisill, M. E., Garcia, C., \& Garcia, L. E. (2008). A Developmental Perspective on the Role of Motor Skill Competence in Physical Activity: An Emergent Relation. Quest, 60(2), 290-306. https://doi.org/10.108/00336297.2008.10483582

Thomas, J. R., Nelson, \&., Silverman, S. (2015). Research Methods in Physical Activity. (7ª ed.), Champaign, IL: Human Kinetics Publishers.

Urich, D. A. (2000). Test of Gross Development, 2nd edition: Examiner’s Manual (2nd ed.). Austin: PRO-ED.

Valentini, N. C. (2012). Validity and reliability of the TGMD-2 for Brazilian children. Journal of Motor Behavior, 44(4), 275-280. https://doi.org/10.3200/JMBR.41.4.357-366

Valentini, N. C., de Almeida, C. S., \& Smith, B. A. (2020). Effectiveness of a home-based early cognitive-motor intervention provided in daycare, home care, and foster care settings: Changes in motor development and context affordances. Early Human Development, Dec; 151:105223. https://doi.org/10.1016/j.earlhumdev.2020.105223

Vandorpe, B., Vandendriessche, J., Vaeyens, R., Pion, J., Matthys, S., Lefevre, J., \& Lenoir, M. (2012). Relationship between sports participation and the level of motor coordination in childhood: A longitudinal approach. Journal of Science and Medicine in Sport, 15(3), 220-225. https://doi.org/10.1016/j.jsams.2011.09.006

Webster, E. K., Martin, C. K., \& Staiano, A. E. (2019). Fundamental motor skills, screen-time, and physical activity in preschoolers. Journal of Sport and Health Science, 8(2), 114-121. https://doi.org/10.1016/j.jshs.2018.11.006

World Heath Organization. (2007). WHO reference 2007. WHO website: http://www.who.int/growthref/who2007_bmi_for_age/en/ 